\title{
Estudo do branqueamento de óleo de peixe utilizando-se a bentonita sódica através de planejamento fatorial $2^{4}$
}

\author{
Study of fish oil bleaching using the sodium bentonite through a \\ factorial design $2^{4}$
}

\author{
Caio Guilherme Secco Souza ${ }^{1}$; Reinaldo Aparecido Bariccatti ${ }^{2}$; Marcia Regina \\ Simões $^{3}$; José Dilson Silva de Oliveira ${ }^{4}$
}

\section{Resumo}

Neste trabalho foi realizado um planejamento experimental $2^{4}$, com o objetivo de otimizar o processo de branqueamento de óleo de peixe, utilizando-se da bentonita sódica como adsorvente, a partir da influência das variáveis : massa de bentonita sódica, temperatura do processo, ativação térmica da bentonita sódica e tempo de agitação. Com isso foi possível adequar um modelo matemático com a influência de cada variável no processo de branqueamento do óleo. A variável resposta foi a absorbância em $444 \mathrm{~nm}$.

Palavras-chave: tilápia. bentonita sódica. adsorção. purificação.

\begin{abstract}
This work was a $2^{4}$ experimental design in order to optimize the process of bleaching of fish oil, using the sodium bentonite as adsorbent, from the influence of variables such as weight of sodium bentonite, the temperature process, thermal activation of sodium bentonite and time of agitation. It was then possible to fit a mathematical model with the influence of each variable in the process of bleaching of the oil. The response variable was the absorbance at $444 \mathrm{~nm}$.
\end{abstract}

Key words: tilápia. sodium bentonite. adsorption. purification.

\section{Introdução}

Atualmente, há uma busca incessante por processos de purificação de diversos tipos de compostos, como óleos animais, vegetais, derivados de petróleo, entre outros, visando redução do tempo e custo de purificação e melhorando suas propriedades físicas, químicas e nutricionais. Esta busca, objetiva o aumento do valor agregado e, consequentemente, maior lucro sobre o produto impuro. Mais especificamente, o óleo vegetal e animal necessitam de processos de purificação para que sejam utilizados na alimentação animal (VIDOTTI; GONÇALVES, 2006), na fabricação de produtos de limpeza como o sabão, ou mesmo como fonte de recursos energéticos, como o biodiesel.

Dentre as etapas de purificação de óleo encontrase a clarificação ou branqueamento (MORAIS et al., 2001), que serve para retirar os constituintes que dão coloração intensa ao óleo. Na purificação de óleos animais e vegetais a etapa de branqueamento, geralmente, é feita com a utilização de agentes adsorventes. Este processo pode ser físico ou

\footnotetext{
Instituto de Química de São Carlos - USP - CEP 13560-970 - São Carlos - SP - Brasil.

2 Centro de Engenharias e Ciências Exatas - Unioeste - CEP 85903-000 - Toledo - PR - Brasil.

3 Centro de Engenharias e Ciências Exatas - Unioeste - CEP 85903 - 000 - Toledo - PR - Brasil

4 Centro de Engenharias e Ciências Exatas - Unioeste - CEP 85903-000 - Toledo - PR - Brasil.
} 
químico e consiste na adesão das moléculas que dão coloração intensa ao óleo numa superfície sólida denominada adsorvente.

Existem alguns tipos de adsorventes utilizados em processos industriais e laboratoriais, e entre estes estão principalmente o carvão ativado, a sílica e os zeólitos. Estes adsorventes são utilizados devido ao seu baixo custo e capacidade de remoção de materiais indesejáveis.

Dentre os zeólitos temos a bentonita sódica, utilizada na clarificação do vinho (CORAZZA; RODRIGUES; NOZAKI, 2001), na ração animal (LOPES et al., 2006), na clarificação de óleo, peneiras moleculares, na indústria de tintas e de cosméticos, adsorção de íons (SANTOS et al., 2002). Sua ampla aplicação industrial está relacionada ao alto poder de inchamento, cerca de 20 vezes seu volume inicial, aumentando as distâncias interplanares até cerca de $100 \AA$, com área superficial entre 30 e 76 $\mathrm{m}^{2} / \mathrm{g}$ (ROSSETTO et al., 2009; NEUMANN et al., 2002).

A utilização deste argilomineral como adsorvente na purificação do óleo animal deve ser estudada para se verificar quais variáveis podem influenciar na eficácia da adsorção das impurezas do óleo de origem animal. Para isto, foi necessário utilizar um planejamento experimental (BARROS; SCARMINIO; BRUNS, 1995; TEÓFILO; FERREIRA, 2006); com as possíveis variáveis que podem influenciar no branqueamento do óleo. As variáveis estudadas pelo planejamento fatorial 24 , são: massa de bentonita $(0,5 \mathrm{~g}$ e $1,0 \mathrm{~g})$, temperatura do processo $\left(30^{\circ} \mathrm{C}\right.$ e $\left.50^{\circ} \mathrm{C}\right)$, ativação térmica da bentonita (não-ativada e ativada) e tempo de agitação (10min e 30min). Este planejamento foi utilizado para otimizar a adsorção das impurezas e determinar quais variáveis possuem efeitos significativos na purificação ou branqueamento? do óleo de peixe, procedimento já utilizado para otimização das condições de biosorção de $\mathrm{Cu}$ (II) (BRASIL et al., 2007).

\section{Materiais e métodos}

Foram pesadas 100 gramas de bentonita sódica, as quais posteriormente foram submetidas a uma temperatura de $200^{\circ} \mathrm{C}$ durante aproximadamente 12 horas, para uma dessorção de substâncias presentes em sua superfície. Tomaram 8 tubos de ensaios e a cada um adicionou-se cerca de $0,5 \mathrm{~g}$ de bentonita (destes, 4 foram de bentonita ativada termicamente e 4 de bentonita não ativada). $\mathrm{O}$ mesmo procedimento foi executado com aproximadamente $1,0 \mathrm{~g}$ de bentonita, totalizando 16 tubos. Para cada tubo foram adicionados $7 \mathrm{~mL}$ de óleo do peixe tilápia (Oreochromis niloticus), e procedeu-se à sua agitação numa incubadora shaker ALPAX . Esta etapa foi feita em duas partes; na primeira foram submetidos à agitação a uma temperatura de $30^{\circ} \mathrm{C}$ e, na segunda, foram agitados a $50^{\circ} \mathrm{C}$. A agitação foi realizada com tempo de 15 minutos e 30 minutos, respectivamente. Este procedimento gerou a matriz de planejamento mostrada na Tabela I.

Posteriormente à agitação, foi realizada a separação entre óleo e bentonita contidos em cada experimento, para a qual foi utilizada a centrifuga HERMLE Z 200 A, durante 5 minutos, a 4000 rpm. Após a centrifugação transferiu-se o líquido sobrenadante de cada experimento para tubos de ensaio correspondente a cada amostra.

Para obtenção do espectro de absorção na região do UV/Vis foi realizada uma diluição por massa, do óleo clarificado em n-hexano, no qual foram pesadas a massas de aproximadamente $0,5 \mathrm{~g}$ do óleo clarificado em cada experimento, em $5 \mathrm{~mL}$ de n-hexano. Após a diluição, realizouse a leitura dos máximos de absorbâncias para cada experimento no espectrofotômetro Shimadzu modelo 1601PC, na região de 200 a $600 \mathrm{~nm}$. Após a varredura de comprimento de onda, verificou-se um máximo característico em $444 \mathrm{~nm}$ para todos os experimentos.

Depois de registradas as absorbâncias, analisaram-se estatisticamente os dados para se verificar a confiabilidade dos resultados e os efeitos 
das variáveis analisadas (massa de bentonita, temperatura, ativação térmica e tempo de agitação) em relação à variável resposta (absorbância em 444 $\mathrm{nm})$. Neste estudos foram empregados os softwares Statistica 7.0 para cálculo da análise de variância (ANOVA) a $5 \%$ de probabilidade e obtiveram as superfícies de resposta.

\section{Resultados e discussão}

A variável resposta foi obtida a partir da varredura de comprimentos de onda realizada no espectrofotômetro, como ilustra a Figura 1. Escolheu a intensidade no máximo de absorção (444nm) para a resposta correspondendo à concentração de impureza, pois em resultados anteriores esta absorbância era totalmente reduzida ao se purificar o óleo.

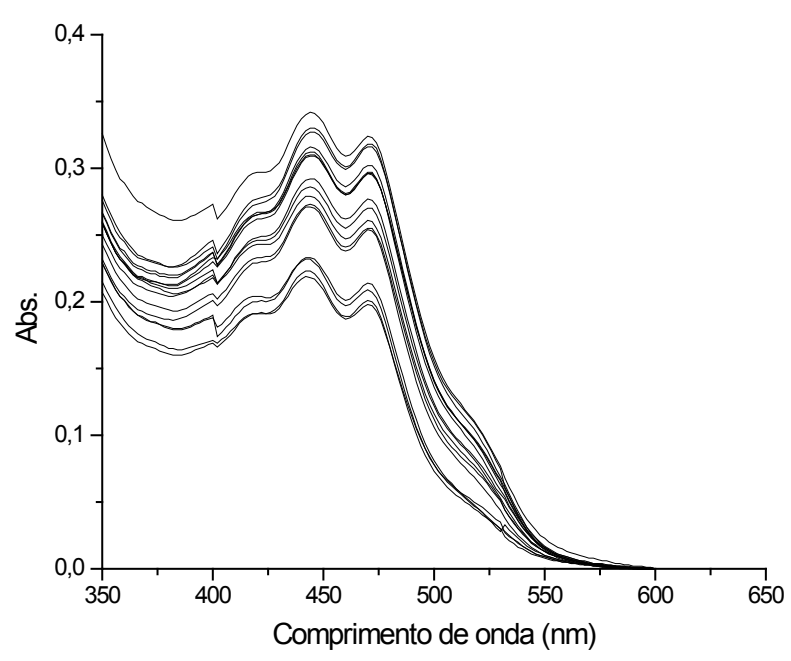

Figura 1. Absorbâncias características das amostras, na região de 350 a 650 nm.

Na Figura 2 mostra-se uma amostra do óleo de peixe antes de ser clarificada pela bentonita (tubo individual) e um conjunto de tubos com os respectivos números da solução, para os quais foi utilizado o processo de purificação.
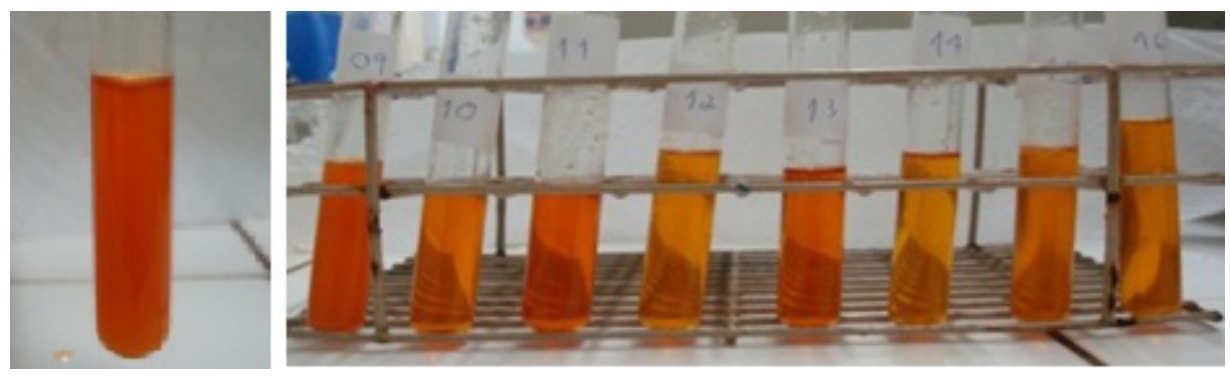

Figura 2. Amostra bruta do óleo de peixe (tubo à esquerda) e conjunto de tubos com o óleo de peixe após o processo de purificação. 
De acordo com a comparação entre os tubos mostrados na Figura 2 (óleo bruto e tratado), percebe-se que houve uma clarificação das amostras de óleo após a utilização da bentonitacomo adsorvente, conferindo diferentes tonalidades às soluções. Dentre as que apresentaram maior clarificação se encontram as soluções $12,14,15$ e 16 e as que apresentaram menor clarificação são as soluções 1, 3 e 5. Estudos realizados por Huang e Sathivel (2010) mostraram que outros adsorventes, como a quitosana, podem purificar e clarificar o óleo de peixe. Estes resultados podem ser quantificados por meio dos espectros mostrados na Figura 1, em que se destaca o agrupamento dos espectros em grupos distintos, com diferentes absorbâncias (de três a quatro grupos), indicando que no mínimo três variáveis ou interações influenciam a resposta.

Os experimentos deste planejamento experimental foram feitos a partir da matriz de planejamento descrita na Tabela I. Nesta também temos a absorbância obtida em 444nm que esta sendo utilizado como variável resposta, a escolha desta variável como resposta considera que a determinação da absorbância no máximo espectral possui um erro menor que daquele obtido no aumento ou redução da absorbância e o efeito de branqueamento é observado pela redução na absorbância na região do visível.

Tabela 1. Matriz de planejamento experimental com a solução (Sol), massa adsorvente (MA), temperatura do processo (TP), ativação do adsorvente (AT), tempo de adsorção (TE) e a absorbância obtida em 444nm (ABS).

\begin{tabular}{llllll} 
Sol & $\mathbf{M A}(\mathbf{g})$ & $\mathbf{T P}\left({ }^{\circ} \mathbf{C}\right)$ & $\mathbf{A T}$ & $\mathbf{T E}(\mathbf{m i n})$ & $\mathbf{A B S}$ \\
1 & 0,5 & 30 & $\mathrm{~N}$ & 10 & 0,327 \\
2 & 1,0 & 30 & $\mathrm{~N}$ & 10 & 0,316 \\
3 & 0,5 & 50 & $\mathrm{~N}$ & 10 & 0,330 \\
4 & 1,0 & 50 & $\mathrm{~N}$ & 10 & 0,312 \\
5 & 0,5 & 30 & $\mathrm{~S}$ & 10 & 0,342 \\
6 & 1,0 & 30 & $\mathrm{~S}$ & 10 & 0,292 \\
7 & 0,5 & 50 & $\mathrm{~S}$ & 10 & 0,309 \\
8 & 1,0 & 50 & $\mathrm{~S}$ & 10 & 0,279 \\
9 & 0,5 & 30 & $\mathrm{~N}$ & 30 & 0,310 \\
10 & 1 & $\mathrm{~N}$ & 30 & 0,271 \\
11 & 0,5 & 50 & $\mathrm{~N}$ & 30 & 0,286 \\
12 & 1,0 & 50 & $\mathrm{~N}$ & 30 & 0,232 \\
13 & 0,5 & 50 & $\mathrm{~S}$ & 30 & 0,273 \\
14 & 1,0 & 30 & $\mathrm{~S}$ & 30 & 0,223 \\
15 & 0,5 & 50 & $\mathrm{~S}$ & 30 & 0,233 \\
16 & 1,0 & 50 & & & 0,218 \\
\hline
\end{tabular}

Como mostrado na Tabela I, foi realizado um planejamento experimental 24, sem experimentos no ponto central, pois um dos fatores (ativação térmica da bentonita) não tem níveis quantitativos, impossibilitando a análise no ponto central.
A partir dos dados obtidos da matriz de planejamento experimental foram analisados os efeitos de cada variável e das interações entre estas. Esta análise encontra-se na Tabela II. 
Tabela 2. Análise do efeito, desvio padrão e significância estatística (p-valor) para os fatores analisados.

\begin{tabular}{llll}
\hline & Efeito & Erro Padrão & p-valor \\
\hline Média & 0,28456 & 0,00361 & 0,00000 \\
Massa (MA) & $-0,03338$ & 0,00723 & 0,00575 \\
Temperatura (TP) & $-0,01938$ & 0,00723 & 0,04379 \\
Ativação (AT) & $-0,02688$ & 0,00723 & 0,01374 \\
Tempo (TE) & $-0,05763$ & 0,00723 & 0,00050 \\
Interação MA:TP & 0,00413 & 0,00723 & 0,59288 \\
Interação MA:AT & $-0,00288$ & 0,00723 & 0,70721 \\
Interação MA:TE & $-0,00613$ & 0,00723 & 0,43543 \\
Interação TP:AT & $-0,00338$ & 0,00723 & 0,660177 \\
Interação TP:TE & $-0,00763$ & 0,00723 & 0,339730 \\
Interação AT:TE & $-0,01113$ & 0,00723 & 0,184375 \\
\hline
\end{tabular}

De acordo com a análise do p-valor na Tabela 2, é possível afirmar que para os fatores principais, massa, temperatura, ativação e tempo, os efeitos são significativos, pois seus respectivos p-valores são menores que 0,05 (o intervalo de confiança para esta análise é de 95\%), o que não acontece com as interações, pois apresentam p-valores maiores que 0,05, o que ocasiona uma não significância para seus efeitos.

De acordo com a Tabela II também observase que os fatores massa de bentonita, temperatura do processo, ativação térmica da bentonita e tempo apresentaram efeitos negativos na variável resposta, o que significa que um aumento nestes fatores (maior massa, maior temperatura, bentonita ativada e maior tempo) causa uma diminuição da absorbância do óleo de peixe (variável resposta), melhorando a clarificação do óleo analisado.

Após a eliminação dos efeitos não significativos (considerados como resíduo), foi verificada por intermédio da Análise de Variância (ANOVA) a significância da regressão a 5\%, utilizando-se do teste F para o planejamento em estudo. A Tabela III representa a ANOVA para este planejamento.

Tabela 3. Análise de Variância (ANOVA) para o branqueamento de óleo de peixe.

\begin{tabular}{llllllc} 
& SQ & GL & MQ & Fcalc. & Ftab. (4,11) & $\mathbf{R}^{\mathbf{2}}$ \\
Regressão & 0,02212 & 4 & 0,00553 & 29,41 & 3,36 & 0,914 \\
Resíduo & 0,00207 & 11 & 0,00019 & & & \\
Total & 0,02419 & 15 & & & & \\
\hline
\end{tabular}

A partir do valores apresentados na Tabela III, constata-se que o modelo apresentou regressão bastante significativa (BARROS; SCARMINIO; BRUNS, 1995), pois Fcalc. $(29,409)$ é maior que o Ftab. (3,36).

O coeficiente de determinação (R2) obtido foi de 0,914 , mostrando que os dados se ajusta muito bem ao modelo proposto.
A partir da influência de cada variável analisada sobre a variável resposta, obtêm-se teoricamente superfícies que relacionam duas variáveis com a variável resposta, com uma representação tridimensional.

Os gráficos da Figura 3 representam as diferentes combinações das variáveis para formar a superfície de resposta. 

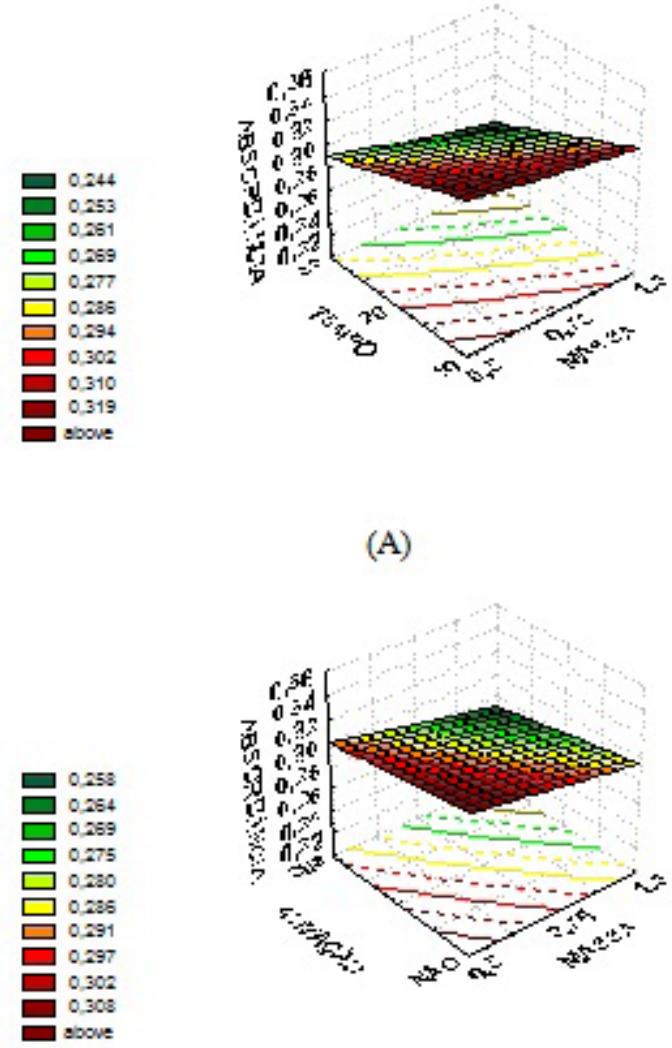

(B)

Figura 3. (A) Superfície de resposta da absorbância em função do tempo de agitação (min.) e da massa de bentonita (g); (B) Superfície de resposta da absorbância em função da ativação térmica da bentonita e da massa de bentonita (g).

Observa-se na Figura de 3 que os gráficos estão inclinados no sentido da diminuição da absorbância quando se tem um aumento quantitativo das variáveis independentes. As inclinações das superfícies são maiores quando influenciadas pela variável tempo, devido ao seu maior efeito na mudança da absorbância, podendo ser evidenciado pelos valores de efeito na Tabela II.

De acordo com os coeficientes de regressão para a matriz de planejamento experimental (Tabela I), foi possível construir um modelo estatístico de primeira ordem para a variável resposta absorbância, em função dos coeficientes de regressão para a média e para cada variável analisada.

Como o modelo linear foi compatível para este planejamento, não foi preciso adicionar pontos axiais na matriz de planejamento. A Tabela IV mostra os coeficientes, também chamados de parâmetros $\beta 0$, $\beta 1, \beta 2, \beta 3$ e $\beta 4$, para a média, massa de bentonita, temperatura do processo, ativação térmica da bentonita e tempo de agitação, respectivamente. Os coeficientes para as interações entre as variáveis não são mostrados na Tabela IV, pois as interações não são significativas a 5\% de significância. Estes coeficientes foram obtidos a partir dos coeficientes de regressão linear. 
Tabela 4. Coeficientes do modelo estatístico para a absorbância.

\begin{tabular}{ll}
\hline Coeficientes & Absorbância \\
\hline$\beta 0$ & 0,351842 \\
$\beta 1$ & $-0,000327$ \\
$\beta 2$ & $-0,000190$ \\
$\beta 3$ & $-0,000263$ \\
$\beta 4$ & $-0,000565$ \\
\hline
\end{tabular}

A partir dos parâmetros da Tabela IV foi possível demonstrar o ajuste do modelo linear pela Equação 1 , com os respectivos valores de $\beta 0, \beta 1, \beta 2, \beta 3$, $\beta 4$ substituídos na equação 1 , sendo que $e$ é o erro experimental.

$\mathrm{y}=0,351842-3,27 \cdot 10^{-4} \mathrm{X}_{1}-1,90 \cdot 10^{-4} \mathrm{X}_{2}-2,63 \cdot 10^{-4} \mathrm{X}_{3}-$ $5,65 \cdot 10^{-4} \mathrm{X}_{4}+e(1)$

Na equação 1 , y é a variável resposta em análise (absorbância), e $X_{1}, X_{2}, X_{3}$ e $X_{4}$ são as variáveis referentes à massa de bentonita, temperatura do processo, ativação térmica da bentonita e tempo de agitação, respectivamente. A partir desta equação pode ser demonstrada a relação que as variáveis analisadas neste planejamento tem com a clarificação do óleo de peixe.

\section{Conclusões}

Constatou-se que todas as variáveis analisadas (massa de bentonita, temperatura do processo, ativação térmica da bentonita e tempo de agitação) foram significativas a 5\% de significância para o processo de branqueamento de óleo de peixe, utilizando-se a bentonita sódica como adsorvente. Porém, as interações entre todas estas variáveis não são significativas para o modelo nestes níveis estudados.

Entre todas as variáveis a que apresentou maior efeito na variável resposta (absorbância) foi o tempo (TE) de agitação, e a variável que apresentou menor efeito foi a temperatura do processo (TP). Porém, com relação a esta última, pode ser possível aumentar seu efeito com um aumento na diferença de temperatura entre os níveis.

O processo de branqueamento de óleo de peixe pode ser otimizado pela influência das variáveis independentes analisadas neste planejamento, em que os maiores níveis $\left(1,0 \mathrm{~g}\right.$ para massa, $50^{\circ} \mathrm{C}$ para temperatura, com ativação térmica da bentonita e 30 minutos de agitação) para todas as variáveis foram os que apresentaram melhores resultados com relação ao branqueamento do óleo.

\section{Referências}

BARROS, B. N.; SCARMINIO, I. S.; BRUNS, R. E. Planejamento e Otimização de Experimentos. Campinas: UNICAMP, 1995.

BRASIL, J. L.; VAGHETTI, C. P.; ROYER, B.; SANTOS JUNIOR, A. A.; SIMON, N. M.; PAVAN, F. A.; DIAS, L. P.; LIMA, E. C. Planejamento estatístico de experimentos como uma ferramenta para otimização das condições de biossorção de $\mathrm{Cu}$ (II) em batelada utilizando-se casca de nozes pecã como biossorvente. Química Nova, São Paulo, v. 30, n. 3, p. 548-553, 2007.

CORAZZA, M. L.; RODRIGUES, D. G.; NOZAKI, J. Preparação e caracterização do vinho de laranja. Química Nova, São Paulo, v. 24, n. 4, p. 449-452, 2001.

HUANG; J.; SATHIVEL, S. Purifying salmon oil using adsorption, neutralization and a combined Neutralization and adsorption process. Journal of Food Engineering, Essex, v. 96, p. 51-58, 2010.

LOPES, J. M.; RUTZ, F.; MALLMANN, C. A.; TOLEDO, G. S. P. Adição de bentonita sódica como adsorvente de aflatoxinas em rações de frangos de corte. Ciência Rural, Santa Maria, v. 36, n. 5, p. 1594-1599, 2006. 
MORAIS, M. M; PINTO, L. A. A.; ORTIZ, S. C. A.; CREXI, V. T; SILVA, R. L.; SILVA, J. D. Estudo do processo de refino do óleo de pescado. Revista do Instituto Adolfo Lutz, São Paulo, v. 60, n. 1, p. 23-33, 2001.

NEUMANN, R.; ALCOVER, A. N.; ARANHA, I. B.; OLIVEIRA, C. H.; LUZ, A. B. Caracterização mineralógica de bentonitas brasileiras. In: ENCONTRO NACIONAL DE TRATAMENTO DE MINÉRIOS E METALURGIA, 19., Recife. Anais... Recife: CETEM, 2002.

ROSSETTO, E.; BERALDIN, R.; PENHA, F. G.; PERGHER, S. B. C. Caracterização de argilas bentonitas e diatomitas e sua aplicação como adsorventes. Química Nova, São Paulo, v. 32, n. 8, p. 2064-2067, 2009.

SANTOS, C. P. F.; MELO, D. M. A.; MELO, M. A. F.; SOBRINHO, E. V. Caracterização e usos de argilas bentonitas e vermiculitas para adsorção de cobre (II) em solução. Cerâmica, São Paulo, v. 48, n. 308, p. 178-182, 2002 .

TEÓFILO, R. F.; FERREIRA, M. M. C. Quimiometria II: planilhas eletrônica para cálculos de planejamentos experimentais, um tutorial. Química Nova, São Paulo, v. 29, n. 2, p. 338-350, 2006.

VIDOTTI, R. M.; GONÇALVES, G. S. Produção e caracterização de silagem, farinha e óleo de Tilápia e sua utilização na alimentação animal. 2006. Disponível em: $<$ ftp://ftp.sp.gov.br/ftppesca/producao_caracterizacao. pdf $>$. Acesso em: 2 maio 2010.

Recebido em 22 Junho 2010-Received on June 22, 2010. Aceito em 28 Fevereiro, 2011 - Accepted on February 28, 2011. 